\title{
Symmetry and Nonexistence of Positive Solutions for Weighted HLS System of Integral Equations on a Half Space
}

\author{
Linfen $\mathrm{Cao}^{1}$ and Zhaohui Dai ${ }^{2}$ \\ ${ }^{1}$ College of Mathematics and Information Science, Henan Normal University, Xinxiang, Henan 453007, China \\ ${ }^{2}$ Department of Computer Science, Henan Normal University, Xinxiang, Henan 453007, China
}

Correspondence should be addressed to Linfen Cao; caolf2010@yahoo.com

Received 28 January 2014; Revised 15 April 2014; Accepted 15 April 2014; Published 30 April 2014

Academic Editor: Bingwen Liu

Copyright (C) 2014 L. Cao and Z. Dai. This is an open access article distributed under the Creative Commons Attribution License, which permits unrestricted use, distribution, and reproduction in any medium, provided the original work is properly cited.

\begin{abstract}
We consider system of integral equations related to the weighted Hardy-Littlewood-Sobolev (HLS) inequality in a half space. By the Pohozaev type identity in integral form, we present a Liouville type theorem when the system is in both supercritical and subcritical cases under some integrability conditions. Ruling out these nonexistence results, we also discuss the positive solutions of the integral system in critical case. By the method of moving planes, we show that a pair of positive solutions to such system is rotationally symmetric about $x_{n}$-axis, which is much more general than the main result of Zhuo and Li, 2011.
\end{abstract}

\section{Introduction}

In [1], Jin and Li studied the weighted HLS system of nonlinear equations in $R^{n}$ :

$$
\begin{aligned}
& u(x)=\frac{1}{|x|^{\alpha}} \int_{R^{n}} \frac{1}{|x-y|^{\lambda}} \frac{v^{q}(y)}{|y|^{\beta}} d y, \\
& v(x)=\frac{1}{|x|^{\beta}} \int_{R^{n}} \frac{1}{|x-y|^{\lambda}} \frac{u^{p}(y)}{|y|^{\alpha}} d y,
\end{aligned}
$$

where $0<\lambda<n$ and $1 /(p+1)+1 /(q+1)=(\lambda+\alpha+\beta) / n$.

By the method of moving planes in integral forms they derived symmetry and monotonicity of positive solutions of (1) under some integrability conditions.

Theorem 1 (see [1]). Let the pair $(u, v)$ be a positive solution of system (1) with $u \in L^{p+1}\left(R^{n}\right), v \in L^{q+1}\left(R^{n}\right)$ and $p, q \geq 1$, $p q \neq 1$, and $\alpha, \beta \geq 0$. Then $u$ and $v$ are radially symmetric and decreasing about some point $x_{0}$.

Jin and $\mathrm{Li} \mathrm{[2]} \mathrm{and} \mathrm{Chen} \mathrm{et} \mathrm{al.} \mathrm{[3]} \mathrm{also} \mathrm{discussed} \mathrm{the} \mathrm{regu-}$ larity of solutions to (1).

Let $R_{+}^{n}$ be the upper half Euclidean space

$$
R_{+}^{n}=\left\{x=\left(x_{1}, \ldots, x_{n}\right) \in R^{n} \mid x_{n}>0\right\} .
$$

In this paper, we want to consider the similar integral system in the half space $R_{+}^{n}$ as (1). More precisely, we discuss the following weighted HLS type system of nonlinear equations in $R_{+}^{n}$ :

$$
\begin{aligned}
& u(x)=\frac{1}{|x|^{\alpha}} \int_{R_{+}^{n}} G(x, y, \gamma) \frac{v^{q}(y)}{|y|^{\beta}} d y, \\
& v(x)=\frac{1}{|x|^{\beta}} \int_{R_{+}^{n}} G(x, y, \gamma) \frac{u^{p}(y)}{|y|^{\alpha}} d y,
\end{aligned}
$$

where $u, v \geq 0,0<p, q<\infty, 0<\gamma<n, \alpha+\beta \geq 0$, $\alpha / n<1 /(p+1)<(n-\gamma+\alpha) / n$, and

$$
G(x, y, \gamma)=\frac{1}{|x-y|^{n-\gamma}}-\frac{1}{\left|x^{*}-y\right|^{n-\gamma}}
$$

here $x^{*}$ is the reflection point of $x$ about the plane $\partial R_{+}^{n}$.

Similar to some integral systems or PDEs systems, the integral system (3) is usually divided into three cases according to the value of exponents $(p, q)$. We say that system (3) is in critical case when the pair $(p, q)$ satisfies the relation

$$
\frac{1}{p+1}+\frac{1}{q+1}=\frac{n-\gamma+\alpha+\beta}{n} \text {. }
$$


It is in supercritical case when " $<$ " holds; and in subcritical case when ">" holds; that is

$$
\frac{1}{p+1}+\frac{1}{q+1}>\frac{n-\gamma+\alpha+\beta}{n} .
$$

In the special case, where $\alpha=0$ and $\beta=0$, system (3) reduces to

$$
\begin{aligned}
& u(x)=\int_{R_{+}^{n}} G(x, y, \gamma) v^{q}(y) d y, \\
& v(x)=\int_{R_{+}^{n}} G(x, y, \gamma) u^{p}(y) d y,
\end{aligned}
$$

and system (7) is closely related to the following system of PDEs with Navier boundary conditions:

$$
\begin{aligned}
& (-\Delta)^{\gamma / 2} u=v^{q}, \quad \text { in } R_{+}^{n} ; \\
& (-\Delta)^{\gamma / 2} v=u^{p}, \quad \text { in } R_{+}^{n} ; \\
& (-\Delta)^{k} u=0 \quad \text { on } \partial R_{+}^{n} ; \\
& (-\Delta)^{k} v=0, \quad \text { on } \partial R_{+}^{n} ; \\
& k=0,1, \ldots, \frac{\gamma}{2}-1 .
\end{aligned}
$$

In particular, when $\gamma$ is an even number, the authors ([4]) proved the equivalence between the two systems (7) and (8) under some mild growth condition.

Symmetry of solutions to integral system (8) was established by Zhuo and Li [5]. They proved that in critical case $1 /(p+1)+1 /(q+1)=(n-\alpha) / n$, any pair of positive solutions of (7) with $u \in L^{p+1}\left(R_{+}^{n}\right)$ and $v \in L^{q+1}\left(R_{+}^{n}\right)$ is rotationally symmetric about some line parallel to $x_{n}$-axis. Under the same integrability conditions, in [6], we obtained the nonexistence of positive solutions of (7).

The general case is that, for $\alpha \neq 0$ and $\beta \neq 0$ in (3), there are few results concerning symmetry and nonexistence for this doubled weighted system. In this paper, by the Pohozaev type identity in integral form, we present a Liouville type theorem when the system (3) is in both supercritical and subcritical cases under some integrability conditions. Based on these nonexistence results, we discuss the positive solutions of (3) in critical case. By the method of moving planes, we show that a pair of positive solutions to such system is rotationally symmetric about $x_{n}$-axis. To carry on the moving of planes, we explore global features of the integral equations and estimate certain integral norms. This is the essence of the method of moving planes in integral forms. The readers who are interested in the integral system and the applications of this method may consult [7-10] and the references therein.

The paper is organized as follows.

In Section 2, by the Pohozaev type identity in integral forms, we prove the following nonexistence results.

Theorem 2. Suppose that $(u(x), v(x)) \in C^{1}\left(R^{n}\right)$ are nonnegative solutions of (3) with $u \in L^{p+1}\left(R_{+}^{n}\right), v \in L^{q+1}\left(R_{+}^{n}\right)$. (i) If $p$ and $q$ are both supercritical, that is,

$$
\frac{1}{p+1}<\frac{n-\gamma}{2 n}+\frac{\alpha}{n}, \quad \frac{1}{q+1}<\frac{n-\gamma}{2 n}+\frac{\beta}{n}
$$

or

(ii) if $p$ and $q$ are both subcritical, that is,

$$
\begin{aligned}
& \frac{1}{p+1} \in\left(\frac{n-\gamma}{2 n}+\frac{\alpha}{n}, \frac{n-\gamma+\alpha}{n}\right), \\
& \frac{1}{q+1} \in\left(\frac{n-\gamma}{2 n}+\frac{\beta}{n}, \frac{n-\gamma+\beta}{n}\right),
\end{aligned}
$$

then $u \equiv 0$ and $v \equiv 0$.

Based on these results and ruling out cases where there are no solutions, we are only interested in critical case (5). In Section 3, by means of method of moving planes in integral form, we establish rotational symmetry of solutions of (3) in critical case (5) as follows.

Theorem 3. Assume that $u \in L^{p+1}\left(R_{+}^{n}\right), v \in L^{q+1}\left(R_{+}^{n}\right)$ and $p, q$ satisfy (5). If $(u, v)$ is a pair of positive solutions of (3), then $(u, v)$ is rotationally symmetric about $x_{n}$-axis.

Remark 4. When $\alpha=\beta=0$, Theorem 3 is coincident with the result in [5].

\section{Proof of Theorem 2}

In this section we will prove the nonexistence of positive solutions to the weighted HLS type system (3). These nonexistence results, known as Liouville type theorems, are useful in deriving existence, a priori estimate, regularity, and asymptotic analysis of solutions.

A celebrated result of S. I. Pohozaev is known as the Pohozaev identity. This classical result has many consequences, the most immediate one being the nonexistence of nontrivial bounded solutions to PDE. Here we apply the Pohozaev type identity in integral forms to the integral system

(3) (see in $[9,11])$.

For any $\rho \neq 0$, there holds

$u(\rho x)=\frac{1}{|\rho x|^{\alpha}} \int_{R_{+}^{n}}\left(\frac{1}{|\rho x-y|^{n-\gamma}}-\frac{1}{\left|\rho x^{*}-y\right|^{n-\gamma}}\right) \frac{v^{q}(y)}{|y|^{\beta}} d y$.

By an elementary calculation,

$$
\begin{aligned}
& \frac{d\left(|\rho x|^{-\alpha}\right)}{d \rho} \\
& =-\frac{\alpha}{2}|\rho x|^{-\alpha-2} \cdot(2 \rho x \cdot x) \\
& =(-\alpha \rho)|\rho x|^{-\alpha-2}|x|^{2} .
\end{aligned}
$$




$$
\begin{aligned}
& \frac{d\left(|\rho x-y|^{\gamma-n}\right)}{d \rho} \\
& =\frac{\gamma-n}{2}|\rho x-y|^{\gamma-n-2} \\
& \quad \times \frac{d}{d \rho}\left[\left(\rho x_{1}-y_{1}\right)^{2}+\cdots+\left(\rho x_{n}-y_{n}\right)^{2}\right] \\
& =(\gamma-n)|\rho x-y|^{\gamma-n-2} x \cdot(\rho x-y), \\
& \frac{d\left(\left|\rho x^{*}-y\right|^{\gamma-n}\right)}{d \rho} \\
& =\frac{\gamma-n}{2}\left|\rho x^{*}-y\right|^{\gamma-n-2} \\
& \quad \times \frac{d}{d \rho}\left[\left(\rho x_{1}-y_{1}\right)^{2}+\cdots+\left(\rho x_{n-1}-y_{n-1}\right)^{2}\right. \\
& \left.\quad+\left(-\rho x_{n}-y_{n}\right)^{2}\right] \\
& =(\gamma-n)|\rho x-y|^{\gamma-n-2} x^{*} \cdot\left(\rho x^{*}-y\right) .
\end{aligned}
$$

Noting $u \in C^{1}\left(R^{n}\right)$, differentiating both sides of (11) with respect to $\rho$ and letting $\rho=1$, we have

$$
\begin{aligned}
x \cdot \nabla u(x)= & (-\alpha) u(x) \\
+(\gamma-n) \frac{1}{|x|^{\alpha}} \int_{R_{+}^{n}} & {\left[\frac{x \cdot(x-y)}{|x-y|^{n-\gamma+2}}\right.} \\
& \left.-\frac{x^{*} \cdot\left(x^{*}-y\right)}{\left|x^{*}-y\right|^{n-\gamma+2}}\right] \frac{v^{q}(y)}{|y|^{\beta}} d y .
\end{aligned}
$$

Let $B_{r}^{+}(0)=B_{r}(0) \cap R_{+}^{n}$ be the upper half ball in the half space in $R_{+}^{n}$. Multiplying left side of (13) by $u^{p}(x)$ and integrating on $B_{r}^{+}$yields

$$
\begin{aligned}
& \int_{B_{r}^{+}} u^{p}(x)(x \cdot \nabla u(x)) d x \\
& \quad=\frac{1}{p+1} \int_{B_{r}^{+}} x \cdot \nabla\left(u^{p+1}(x)\right) d x \\
& \quad=\frac{1}{p+1} \int_{\partial B_{r}^{+}} r u^{p+1}(x) d \sigma-\frac{n}{p+1} \int_{B_{r}^{+}} u^{p+1}(x) d x .
\end{aligned}
$$

Similarly, we also have

$$
\begin{aligned}
& \int_{B_{r}^{+}} v^{q}(x)(x \cdot \nabla v(x)) d x \\
& \quad=\frac{1}{q+1} \int_{\partial B_{r}^{+}} r v^{q+1}(x) d \sigma-\frac{n}{q+1} \int_{B_{r}^{+}} v^{q+1}(x) d x .
\end{aligned}
$$

\section{Since}

$$
\int_{R_{+}^{n}} u^{p+1}(x) d x<\infty, \quad \int_{R_{+}^{n}} v^{q+1}(x) d x<\infty .
$$

Thus, there exists a sequence $\left\{r_{m}\right\}$ such that

$$
\begin{aligned}
r_{m} \int_{\partial B_{r_{m}}^{+}} u^{p+1}(x) d \sigma & \longrightarrow 0, \\
\int_{\partial B_{r_{m}}^{+}} r_{m} v^{q+1}(x) d \sigma & \longrightarrow 0, \\
r_{m} & \longrightarrow \infty .
\end{aligned}
$$

Let $r_{m} \rightarrow \infty$; by (14), (15), and (17), we have

$$
\begin{aligned}
& \int_{R_{+}^{n}} u^{p}(x)(x \cdot \nabla u(x)) d x+\int_{R_{+}^{n}} v^{q}(x)(x \cdot \nabla v(x)) d x \\
& =-\frac{n}{p+1} \int_{R_{+}^{n}} u^{p+1}(x) d x-\frac{n}{q+1} \int_{R_{+}^{n}} v^{q+1}(x) d x<\infty .
\end{aligned}
$$

On the other hand,

$$
\begin{aligned}
& \int_{R_{+}^{n}} u^{p}(x)(x \cdot \nabla u(x)) d x \\
&=(\gamma-n) \iint_{R_{+}^{n}} {\left[\frac{x \cdot(x-y)}{|x-y|^{n-\gamma+2}}-\frac{x^{*} \cdot\left(x^{*}-y\right)}{\left|x^{*}-y\right|^{n-\gamma+2}}\right] } \\
& \times \frac{u^{p}(x) v^{q}(y)}{|x|^{\alpha}|y|^{\beta}} d x d y \\
&+(-\alpha) \int_{R_{+}^{n}} u^{p+1}(x) d x \\
&=\frac{\gamma-n}{2} \iint_{R_{+}^{n}}\left[\frac{x \cdot(x-y)}{|x-y|^{n-\gamma+2}}-\frac{x^{*} \cdot\left(x^{*}-y\right)}{\left|x^{*}-y\right|^{n-\gamma+2}}\right] \\
& \times \frac{u^{p}(x) v^{q}(y)}{|x|^{\alpha}|y|^{\beta}} d x d y \\
&+\frac{\gamma-n}{2} \int_{R_{+}^{n}}\left[\frac{y \cdot(y-x)}{|x-y|^{n-\gamma+2}}-\frac{y^{*} \cdot\left(y^{*}-x\right)}{\left|y^{*}-x\right|^{n-\gamma+2}}\right] \\
&+(-\alpha) \int_{R_{+}^{n}} u^{p+1}(x) d x . \\
& \times \frac{u^{p}(y) v^{q}(x)}{|y|^{\alpha}|x|^{\beta}} d x d y
\end{aligned}
$$

There also holds

$$
\begin{aligned}
& \int_{R_{+}^{n}} v^{q}(x)(x \cdot \nabla v(x)) d x \\
&=(\gamma-n) \iint_{R_{+}^{n}} {\left[\frac{x \cdot(x-y)}{|x-y|^{n-\gamma+2}}-\frac{x^{*} \cdot\left(x^{*}-y\right)}{\left|x^{*}-y\right|^{n-\gamma+2}}\right] } \\
& \times \frac{u^{p}(y) v^{q}(x)}{|y|^{\alpha}|x|^{\beta}} d x d y \\
&+(-\beta) \int_{R_{+}^{n}} v^{q+1}(x) d x
\end{aligned}
$$




$$
\begin{aligned}
& =\frac{\gamma-n}{2} \iint_{R_{+}^{n}}\left[\frac{x \cdot(x-y)}{|x-y|^{n-y+2}}-\frac{x^{*} \cdot\left(x^{*}-y\right)}{\left|x^{*}-y\right|^{n-\gamma+2}}\right] \\
& \times \frac{u^{p}(y) v^{q}(x)}{|y|^{\alpha}|x|^{\beta}} \\
& +\frac{\gamma-n}{2} \iint_{R_{+}^{n}}\left[\frac{y \cdot(y-x)}{|x-y|^{n-\gamma+2}}-\frac{y^{*} \cdot\left(y^{*}-x\right)}{\left|y^{*}-x\right|^{n-\gamma+2}}\right] \\
& \quad \times \frac{u^{p}(x) v^{q}(y)}{|x|^{\alpha}|y|^{\beta}} d x d y \\
& +(-\beta) \int_{R_{+}^{n}} v^{q+1}(x) d x .
\end{aligned}
$$

Using

$$
\begin{gathered}
x \cdot(x-y)+y \cdot(y-x)=|x-y|^{2}, \\
x^{*} \cdot\left(x^{*}-y\right)+y^{*} \cdot\left(y^{*}-x\right)=\left|x^{*}-y\right|^{2} .
\end{gathered}
$$

Combining the fact $\left|x^{*}-y\right|=\left|y^{*}-x\right|$, (19), and (20), we have

$$
\begin{aligned}
\int_{R_{+}^{n}} u^{p}(x)(x \cdot \nabla u(x)) d x+\int_{R_{+}^{n}} v^{q}(x)(x \cdot \nabla v(x)) d x \\
=\left(\frac{\gamma-n}{2}-\alpha\right) \int_{R_{+}^{n}} u^{p+1}(x) d x \\
\quad+\left(\frac{\gamma-n}{2}-\beta\right) \int_{R_{+}^{n}} v^{q+1}(x) d x .
\end{aligned}
$$

By (18) and (22), we have

$$
\begin{aligned}
& \left(\frac{\gamma-n}{2}-\alpha+\frac{n}{p+1}\right) \int_{R_{+}^{n}} u^{p+1}(x) d x \\
& \quad+\left(\frac{\gamma-n}{2}-\beta+\frac{n}{q+1}\right) \int_{R_{+}^{n}} v^{q+1}(x) d x=0 .
\end{aligned}
$$

Hence, if

$$
\frac{\gamma-n}{2}-\alpha+\frac{n}{p+1}>0, \quad \frac{\gamma-n}{2}-\beta+\frac{n}{q+1}>0
$$

or

$$
\frac{\gamma-n}{2}-\alpha+\frac{n}{p+1}<0, \quad \frac{\gamma-n}{2}-\beta+\frac{n}{q+1}<0,
$$

hold, it follows that $u \equiv 0$ and $v \equiv 0$.

This completes the proof of Theorem 2.

Remark 5. In [11], the authors consider another weighted HLS type integral system

$$
\begin{aligned}
& u(x)=\int_{R_{+}^{n}} G(x, y, \gamma)|y|^{-s} v^{q}(y) d y, \\
& v(x)=\int_{R_{+}^{n}} G(x, y, \gamma)|y|^{-t} u^{p}(y) d y,
\end{aligned}
$$

$$
\forall x \in R_{+}^{n}
$$

and showed the Liouville type theorem as follows.
Theorem 6 (see [11]). Suppose that $u(x), v(x) \in C^{1}\left(R^{n}\right)$ are positive solutions of (26) when $p$ and q are both subcritical; that is $1 /(p+1)>(n-\gamma) / 2(n-t)$ and $1 /(q+1)>(n-\gamma) / 2(n-s)$. If $\int_{R_{+}^{n}}\left(u^{p+1} /|x|^{t}\right) d x<\infty, \int_{R_{+}^{n}}\left(v^{q+1} /|x|^{s}\right) d x<\infty$ and $\gamma-s>1$, $\gamma-t>1$, then $u \equiv 0$ and $v \equiv 0$.

When $s=t=0$ in system (26) or $\alpha=\beta=0$ in system (3), the two systems reduce to the simple integral system (7). In this special case, we can find that Theorem 6 is coincident with case (ii) in Theorem 2.

\section{Proof of Theorem 3}

In this section, we will consider rotational symmetry of weighted HLS type system (3) in critical case (5).

Firstly, we need the following weighted HLS inequality.

Lemma 7 (see [12]). Let $1<l, m<\infty, 0<\gamma<n, \tau+\beta \geq 0$, $1 / l+1 / m+(\gamma+\tau+\beta) / n=2$, and $1-1 / m-\gamma / n<\tau / n<1-1 / m$. Then

$$
\left|\iint_{R^{n}} \frac{f(x) g(y)}{|x|^{\tau}|x-y|^{\gamma}|y|^{\beta}} d x d y\right| \leq C\|f\|_{m}\|g\|_{l} .
$$

One can also write the weighted HLS inequality in another form. Let

$$
\operatorname{Tg}(x)=\int_{R^{n}} \frac{g(y)}{|x|^{\tau}|x-y|^{\gamma}|y|^{\beta}} d y .
$$

Then

$$
\|T g(x)\|_{L^{\mu}}=\operatorname{Sup}_{\|f\|_{m}=1}<T g(x), \quad f(x)>\leq\|g\|_{L^{l}},
$$

where $1 / l+(\gamma+\tau+\beta) / n=1+1 / \mu, 1 / \mu+1 / m=1$.

For a given real number $\lambda$, define

$$
\begin{aligned}
& \Sigma_{\lambda}=\left\{x=\left(x_{1}, x_{2}, \ldots, x_{n}\right) \in R_{+}^{n} \mid x_{1}<\lambda\right\}, \\
& T_{\lambda}=\left\{x \in R_{+}^{n} \mid x_{1}=\lambda\right\} .
\end{aligned}
$$

Let $x^{\lambda}=\left(2 \lambda-x_{1}, x_{2}, \ldots, x_{n-1}, x_{n}\right)$ be the reflection of the point $x=\left(x_{1}, x_{2}, \ldots, x_{n}\right)$ about the plane $T_{\lambda}$. Set

$$
u_{\lambda}(x)=u\left(x^{\lambda}\right), \quad v_{\lambda}(x)=v\left(x^{\lambda}\right)
$$

Lemma 8 (see $[8,13])$. For $x, y \in \Sigma_{\lambda}, x \neq y$, one has

$$
G(x, y, \gamma) \geq G\left(x^{\lambda}, y, \gamma\right)
$$


Lemma 9. Let $(u, v)$ be any pair of positive solutions of (3) in critical case (5); for any $x \in \Sigma_{\lambda}$ and $|x|>\left|x^{\lambda}\right|$, one has

$$
\begin{aligned}
& u(x)-u_{\lambda}(x) \\
& \leq \frac{1}{|x|^{\alpha}} \int_{\Sigma_{\lambda}}\left[G(x, y, \gamma)-G\left(x^{\lambda}, y, \gamma\right)\right] \frac{v^{q}(y)-v_{\lambda}^{q}(y)}{|y|^{\beta}} d y, \\
& v(x)-v_{\lambda}(x) \\
& \leq \frac{1}{|x|^{\beta}} \int_{\Sigma_{\lambda}}\left[G(x, y, \gamma)-G\left(x^{\lambda}, y, \gamma\right)\right] \frac{u^{p}(y)-u_{\lambda}^{p}(y)}{|y|^{\alpha}} d y .
\end{aligned}
$$

Proof. Through the calculation, we have

$$
\begin{aligned}
u(x)= & \frac{1}{|x|^{\alpha}} \int_{\Sigma_{\lambda}} G(x, y, \gamma) \frac{v^{q}(y)}{|y|^{\beta}} d y \\
& +\frac{1}{|x|^{\alpha}} \int_{\Sigma_{\lambda}} G\left(x^{\lambda}, y, \gamma\right) \frac{v_{\lambda}^{q}(y)}{\left|y^{\lambda}\right|^{\beta}} d y, \\
u_{\lambda}(x)= & \frac{1}{\left|x^{\lambda}\right|^{\alpha}} \int_{\Sigma_{\lambda}} G\left(x^{\lambda}, y, \gamma\right) \frac{v^{q}(y)}{|y|^{\beta}} d y \\
& +\frac{1}{\left|x^{\lambda}\right|^{\alpha}} \int_{\Sigma_{\lambda}} G(x, y, \gamma) \frac{u_{\lambda}^{p}(y)}{\left|y^{\lambda}\right|^{\beta}} d y .
\end{aligned}
$$

By the assumption $|x|>\left|x^{\lambda}\right|$, we have

$$
\begin{aligned}
& u(x)-u_{\lambda}(x) \\
& \leq \frac{1}{|x|^{\alpha}} \int_{\Sigma_{\lambda}}\left[G(x, y, \gamma)-G\left(x^{\lambda}, y, \gamma\right)\right] \\
& \quad \times\left(\frac{v^{q}(y)}{|y|^{\beta}}-\frac{v_{\lambda}^{q}(y)}{\left|y^{\lambda}\right|^{\beta}}\right) d y \\
& \leq \frac{1}{|x|^{\alpha}} \int_{\Sigma_{\lambda}}\left[G(x, y, \gamma)-G\left(x^{\lambda}, y, \gamma\right)\right] \frac{v^{q}(y)-v_{\lambda}^{q}(y)}{|y|^{\beta}} d y .
\end{aligned}
$$

Similarly, we have

$$
\begin{gathered}
v(x)-v_{\lambda}(x) \\
\leq \frac{1}{|x|^{\beta}} \int_{\Sigma_{\lambda}}\left[G(x, y, \gamma)-G\left(x^{\lambda}, y, \gamma\right)\right] \\
\times \frac{u^{p}(y)-u_{\lambda}^{p}(y)}{|y|^{\alpha}} d y .
\end{gathered}
$$

Proof of Theorem 3. Step 1. We will show that for sufficiently negative $\lambda$,

$$
u_{\lambda}(x) \geq u(x), \quad v_{\lambda}(x) \geq v(x), \quad \text { a.e. } \forall x \in \Sigma_{\lambda} .
$$

Define

$$
\begin{aligned}
& \Sigma_{\lambda}^{u}=\left\{x \in \Sigma_{\lambda}, u(x)>u_{\lambda}(x)\right\}, \\
& \Sigma_{\lambda}^{v}=\left\{x \in \Sigma_{\lambda}, v(x)>v_{\lambda}(x)\right\} .
\end{aligned}
$$

We prove that, for sufficiently negative $\lambda$, both $\Sigma_{\lambda}^{u}$ and $\Sigma_{\lambda}^{v}$ must be empty and thus (37) holds.

In fact, by Lemma 9 and the mean value theorem, we have, for $x \in \Sigma_{\lambda}^{u}$,

$$
\begin{aligned}
0 & <u(x)-u_{\lambda}(x) \\
& \leq \frac{1}{|x|^{\alpha}} \int_{\Sigma_{\lambda}}\left[G(x, y, \gamma)-G\left(x^{\lambda}, y, \gamma\right)\right] \frac{v^{q}(y)-v_{\lambda}^{q}(y)}{|y|^{\beta}} d y \\
& \leq \int_{\Sigma_{\lambda}^{v}} \frac{1}{|x|^{\alpha}}\left[G(x, y, \gamma)-G\left(x^{\lambda}, y, \gamma\right)\right] \frac{v^{q}(y)-v_{\lambda}^{q}(y)}{|y|^{\beta}} d y \\
& \leq \frac{1}{|x|^{\alpha}} \int_{\Sigma_{\lambda}^{v}} G(x, y, \gamma) \frac{v^{q}(y)-v_{\lambda}^{q}(y)}{|y|^{\beta}} d y \\
& \leq \frac{q}{|x|^{\alpha}} \int_{\Sigma_{\lambda}^{v}} \frac{1}{|x-y|^{n-\gamma}} \psi_{\lambda}^{q-1}(y) \frac{v(y)-v_{\lambda}(y)}{|y|^{\beta}} d y \\
& \leq q \int_{\Sigma_{\lambda}^{v}} \frac{1}{|x|^{\alpha}|x-y|^{n-\gamma}|y|^{\beta}} v^{q-1}(y)\left[v(y)-v_{\lambda}(y)\right] d y,
\end{aligned}
$$

where $\psi_{\lambda}(y)$ is valued between $v(y)$ and $v_{\lambda}(y)$; therefore, on $\Sigma_{\lambda}^{v}$, we have

$$
0 \leq v_{\lambda}(y) \leq \psi_{\lambda}(y) \leq v(y)
$$

By Lemma 7 and the Hölder inequality, we have

$$
\begin{aligned}
\left\|u_{\lambda}-u\right\|_{L^{p+1}\left(\Sigma_{\lambda}^{u}\right)} & \leq C\left\|v^{q-1}\left(v_{\lambda}-v\right)\right\|_{L^{T_{1}}\left(\Sigma_{\lambda}^{v}\right)} \\
& \leq C\|v\|_{L^{q+1}\left(\Sigma_{\lambda}^{v}\right)}^{q-1}\left\|v_{\lambda}-v\right\|_{L^{q+1}\left(\Sigma_{\lambda}^{v}\right)}, \\
\left\|v_{\lambda}-v\right\|_{L^{q+1}\left(\Sigma_{\lambda}^{v}\right)} \leq C\left\|u^{p-1}\left(u_{\lambda}-u\right)\right\|_{L^{T_{2}}\left(\Sigma_{\lambda}^{u}\right)} & \leq C\|u\|_{L^{p+1}\left(\Sigma_{\lambda}^{u}\right)}^{p-1}\left\|u_{\lambda}-u\right\|_{L^{p+1}\left(\Sigma_{\lambda}^{u}\right)},
\end{aligned}
$$

where $T_{1}=n(p+1) /(n+(\gamma-\alpha-\beta)(p+1))$ and $T_{2}=n(q+$ $1) /(n+(\gamma-\alpha-\beta)(q+1))$. It easy to show that $T_{1}, T_{2}>1$. Combining (41) and (42), we arrive

$$
\begin{aligned}
& \left\|u_{\lambda}-u\right\|_{L^{p+1}\left(\Sigma_{\lambda}^{u}\right)} \\
& \quad \leq C\|v\|_{L^{q+1}\left(\Sigma_{\lambda}^{v}\right)}^{q-1}\|u\|_{L^{p+1}\left(\Sigma_{\lambda}^{u}\right)}^{p-1}\left\|u_{\lambda}-u\right\|_{L^{p+1}\left(\Sigma_{\lambda}^{u}\right)} .
\end{aligned}
$$

The conditions $u \in L^{p+1}\left(R_{+}^{n}\right)$ and $v \in L^{q+1}\left(R_{+}^{n}\right)$ make us able to choose sufficiently negative $\lambda$, so that

$$
C\|v\|_{L^{q+1}\left(\Sigma_{\lambda}^{v}\right)}^{q-1}\|u\|_{L^{p+1}\left(\Sigma_{\lambda}^{u}\right)}^{p-1} \leq \frac{1}{2} .
$$


Now inequality (43) implies

$$
\left\|u_{\lambda}-u\right\|_{L^{p+1}\left(\Sigma_{\lambda}^{u}\right)}=0
$$

and therefore $\Sigma_{\lambda}^{u}$ must be measure zero. Similarly, one can show that $\Sigma_{\lambda}^{v}$ is measure zero. Therefore (37) holds.

Step 2. Inequality (37) provides a starting point to move the plane $T_{\lambda}=\left\{x \in R_{+}^{n} \mid x_{1}=\lambda\right\}$. Now we start from the neighborhood of $x_{1}=-\infty$ and move the plane to the right as long as (37) holds to the limiting position. More precisely, define

$$
\begin{aligned}
\lambda_{0}=\sup \{\lambda \mid u(x) & \leq u_{\mu}(x), \\
v(x) & \left.\leq v_{\mu}(x), \mu \leq \lambda, \forall x \in \Sigma_{\mu}\right\} .
\end{aligned}
$$

We will prove that $\lambda_{0}=0$. On the contrary, we suppose $\lambda_{0}<$ 0 . We show that $u(x)$ and $v(x)$ are symmetric about the plane $T_{\lambda_{0}}$; that is

$$
u_{\lambda_{0}}(x) \equiv u(x), \quad v_{\lambda_{0}}(x) \equiv v(x), \quad \text { a.e. } \forall x \in \Sigma_{\lambda_{0}} .
$$

Otherwise, on $\Sigma_{\lambda_{0}}$,

$$
\begin{aligned}
& u(x) \leq u_{\lambda_{0}}(x), \quad v(x) \leq v_{\lambda_{0}}(x), \\
& \text { but } u(x) \not \equiv u_{\lambda_{0}}(x) \quad \text { or } \quad v(x) \not \equiv v_{\lambda_{0}}(x) .
\end{aligned}
$$

We show that the plane can be moved further to the right. More precisely, there exists an $\epsilon>0$ such that, for $\forall \lambda \epsilon$ $\left[\lambda_{o}, \lambda_{o}+\epsilon\right)$,

$$
u(x) \leq u_{\lambda}(x), \quad v(x) \leq v_{\lambda}(x), \quad \text { a.e. } \forall x \in \Sigma_{\lambda} .
$$

Without loss of generality, we assume

$$
v(x) \not \equiv v_{\lambda_{0}}(x), \quad \text { on } \Sigma_{\lambda_{0}} \text {. }
$$

by Lemma 9, we have in fact $u(x)<u_{\lambda_{0}}(x)$ in the interior of $\Sigma_{\lambda_{0}}$. Let

$$
\begin{aligned}
& \overline{\sum_{\lambda_{0}}^{u}}=\left\{x \in \Sigma_{\lambda_{0}} \mid u(x) \geq u_{\lambda_{0}}(x)\right\}, \\
& \overline{\sum_{\lambda_{0}}^{v}}=\left\{x \in \Sigma_{\lambda_{0}} \mid v(x) \geq v_{\lambda_{0}}(x)\right\} .
\end{aligned}
$$

Then obviously $\overline{\Sigma_{\lambda_{0}}^{u}}$ has measure zero and $\lim _{\lambda \rightarrow \lambda_{0}} \Sigma_{\lambda}^{u} \subset \overline{\Sigma_{\lambda_{0}}^{u}}$. The same argument above is also true for the other solution $v$ of (3). From (41) and (42), we deduce

$$
\begin{aligned}
& \left\|u_{\lambda}-u\right\|_{L^{p+1}\left(\Sigma_{\lambda}^{u}\right)} \\
& \quad \leq C\|v\|_{L^{q+1}\left(\Sigma_{\lambda}^{v}\right)}^{q-1}\|u\|_{L^{p+1}\left(\Sigma_{\lambda}^{u}\right)}^{p-1}\left\|u_{\lambda}-u\right\|_{L^{p+1}\left(\Sigma_{\lambda}^{u}\right)} .
\end{aligned}
$$

Again the conditions that $u \in L^{p+1}\left(R_{+}^{n}\right)$ and $v \in L^{q+1}\left(R_{+}^{n}\right)$ ensure that one can choose $\epsilon$ sufficiently small, so that, for all $\lambda$ in $\left[\lambda_{o}, \lambda_{o}+\epsilon\right)$,

$$
C\|v\|_{L^{q+1}\left(\Sigma_{\lambda}^{v}\right)}^{q-1}\|u\|_{L^{p+1}\left(\Sigma_{\lambda}^{u}\right)}^{p-1} \leq \frac{1}{2} .
$$

The method to verify this inequality is standard and the proofs of the rest are similar to the proof in paper $[6,11,14]$.

Now by (52) and (53), we have $\left\|u_{\lambda}-u\right\|_{L^{p}\left(\Sigma_{\lambda}^{u}\right)}=0$, and therefore $\Sigma_{\lambda}^{u}$ must be measure zero. Similarly, $\Sigma_{\lambda}^{v}$ must also be measure zero. Hence, for these values of $\lambda>\lambda_{o}$, we have

$$
u_{\lambda}(x) \geq u(x), \quad v_{\lambda}(x) \geq v(x), \quad \text { a.e. } \forall x \in \Sigma_{\lambda_{0}} .
$$

This (47) must hold and therefore both $u(x)$ and $v(x)$ are symmetric about the plane $T_{\lambda_{0}}$.

Now we show that the plane cannot stop before hitting the origin. Otherwise, assume that the plane stops at $x_{1}=\lambda_{0}<0$. By the fact that $|y|>\left|y^{\lambda_{0}}\right|$, we have

$$
\begin{array}{r}
u(x)-u_{\lambda_{0}}(x) \\
\leq \frac{1}{|x|^{\alpha}} \int_{\Sigma_{\lambda_{0}}}\left[G(x, y, \gamma)-G\left(x^{\lambda_{0}}, y, \gamma\right)\right] \\
\times\left[\frac{v^{q}(y)}{\left.|y|^{\beta}-\frac{v_{\lambda_{0}}^{q}(y)}{\left|y^{\lambda_{0}}\right|^{\beta}}\right] d y}\right. \\
<\frac{1}{|x|^{\alpha}} \int_{\Sigma_{\lambda_{0}}}\left[G(x, y, \gamma)-G\left(x^{\lambda_{0}}, y, \gamma\right)\right] \\
\times\left[\frac{v^{q}(y)-v_{\lambda_{0}}^{q}(y)}{|y|^{\beta}}\right] d y=0 .
\end{array}
$$

This contradicts with (47).

As the direction of $x_{1}$ can be chosen arbitrarily, we derive that $(u(x), v(x))$ is rotationally symmetric about $x_{n}$-axis. This completes the proof of Theorem 3.

\section{Conflict of Interests}

The authors declare that there is no conflict of interests regarding the publication of this paper.

\section{Acknowledgments}

The authors would like to thank Professor Wenxiong Chen and the referees for their valuable suggestions and comments. This work is supported by Grant (nos. U1304101 and 11171091) of NSFC and NSF of Henan Province (no. 132300410141).

\section{References}

[1] C. Jin and C. Li, "Symmetry of solutions to some systems of integral equations," Proceedings of the American Mathematical Society, vol. 134, no. 6, pp. 1661-1670, 2006.

[2] C. Jin and C. Li, "Quantitative analysis of some system of integral equations," Calculus of Variations and Partial Differential Equations, vol. 26, no. 4, pp. 447-457, 2006.

[3] W. Chen, C. Jin, C. Li, and J. Lim, "Weighted Hardy-LittlewoodSobolev inequalities and systems of integral equations," Discrete and Continuous Dynamical Systems A, vol. 2005, supplement, pp. 164-172, 2005. 
[4] W. Zhao, J. Yang, and S. Zheng, "Liouville type theorem for higher-orderelliptic system with Navier boundary condition," 2013.

[5] R. Zhuo and D. Li, "A system of integral equations on half space," Journal of Mathematical Analysis and Applications, vol. 381, no. 1, pp. 392-401, 2011.

[6] L. Cao and Z. Dai, "A Liouville-type theorem for an integral system on a half-space $R_{+}^{n}$," Journal of Inequalities and Applications, vol. 2013, article 37, 2013.

[7] Y. Fang and J. Zhang, "Nonexistence of positive solution for an integral equation on a half-space $R_{+}^{n}$," Communications on Pure and Applied Analysis, vol. 12, no. 2, pp. 663-678, 2013.

[8] L. Cao and W. Chen, "Liouville type theorems for poly-harmonic Navier problems," Discrete and Continuous Dynamical Systems A, vol. 33, no. 9, pp. 3937-3955, 2013.

[9] C. Li and Y. Lei, "Sharp criteria of Liouville thpe for somenonlinear systems," http://arxiv.org/abs/1301.6235.

[10] Y. Zhao, "Regularity and symmetry for solutions to a system of weighted integral equations," Journal of Mathematical Analysis and Applications, vol. 391, no. 1, pp. 209-222, 2012.

[11] D. Li, P. Niu, and R. Zhuo, "Symmetry and non-existence of positive solutions for PDE system with Navier boundary conditions on a half space," Complex Variables and Elliptic Equations.

[12] E. M. Stein and G. Weiss, Introduction to Fourier Analysis on Euclidean Spaces, Princeton University Press, Princeton, NJ, USA, 1971.

[13] D. Li and R. Zhuo, "An integral equation on half space," Proceedings of the American Mathematical Society, vol. 138, no. 8, pp. 2779-2791, 2010.

[14] L. Cao and Z. Dai, "A Liouville-type theorem for an integral equation on a half-space $R_{+}^{n}$," Journal of Mathematical Analysis and Applications, vol. 389, no. 2, pp. 1365-1373, 2012. 


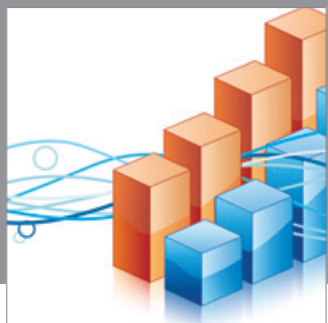

Advances in

Operations Research

mansans

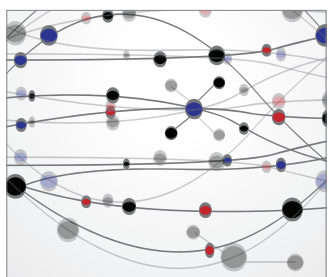

The Scientific World Journal
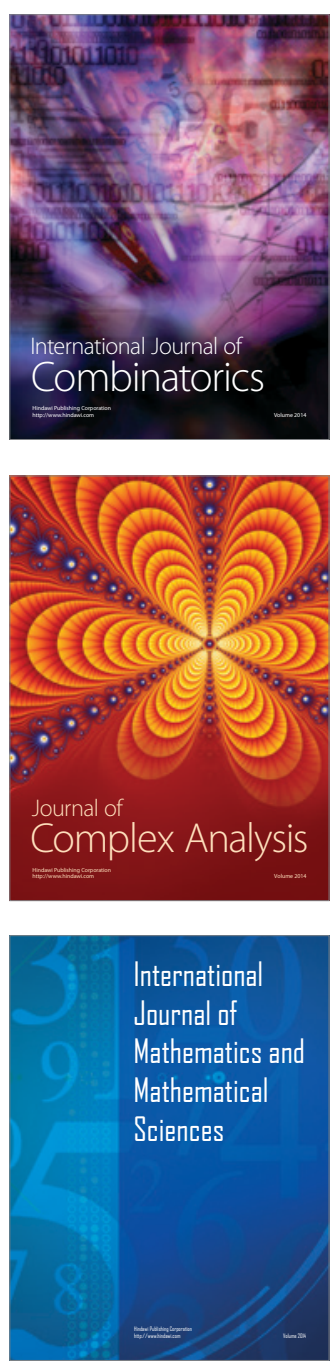
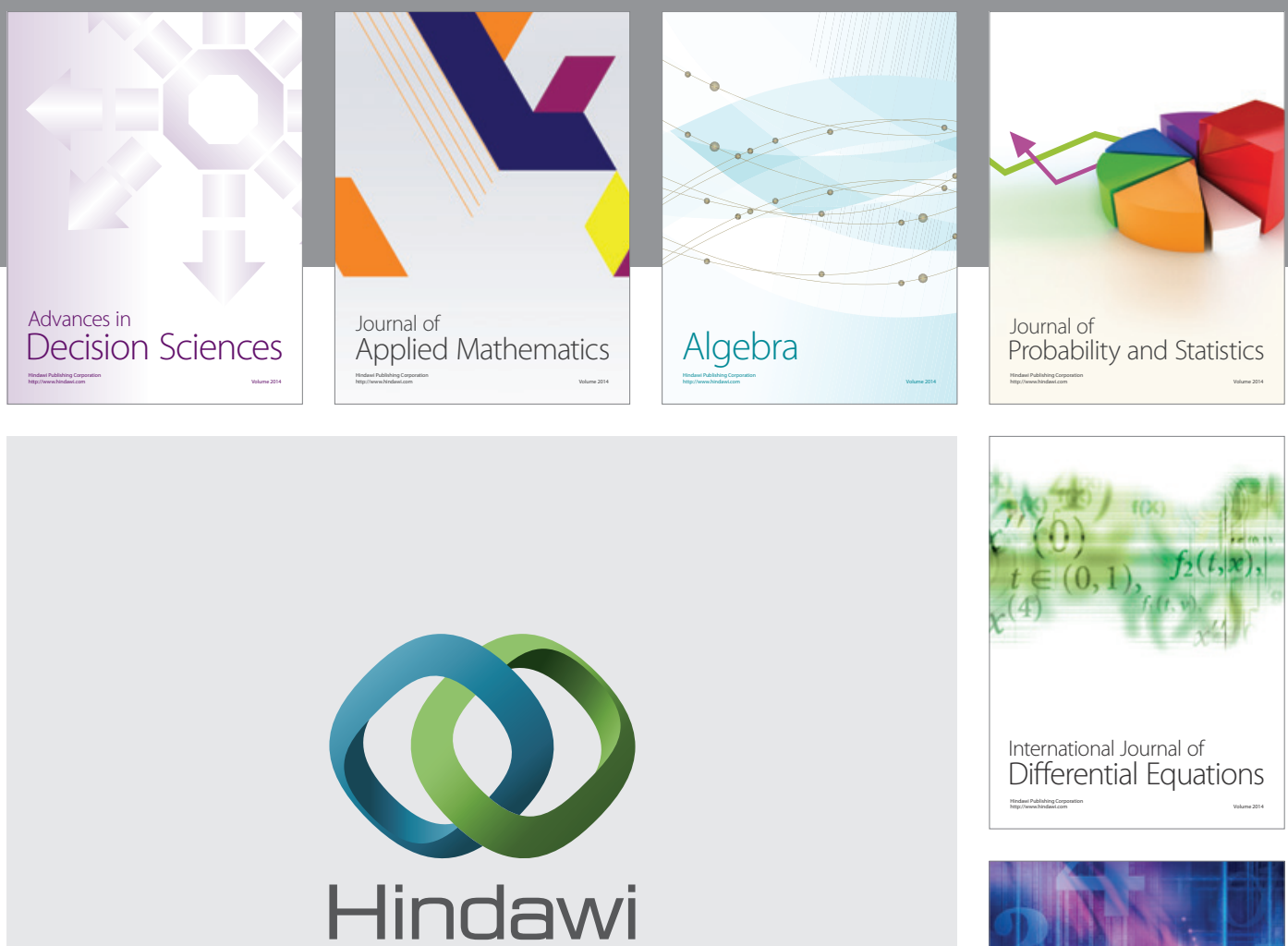

Submit your manuscripts at http://www.hindawi.com
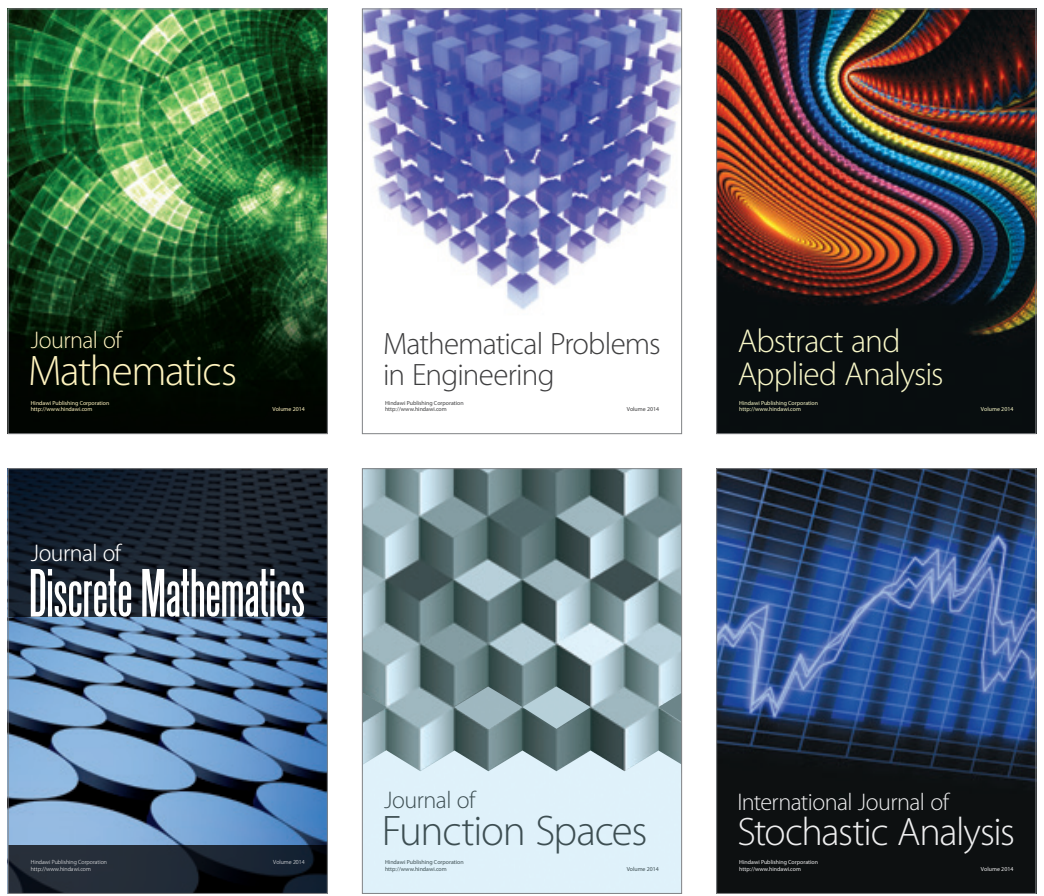

Journal of

Function Spaces

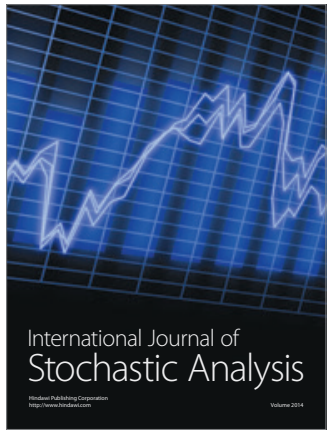

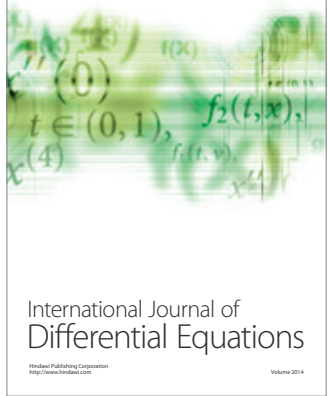
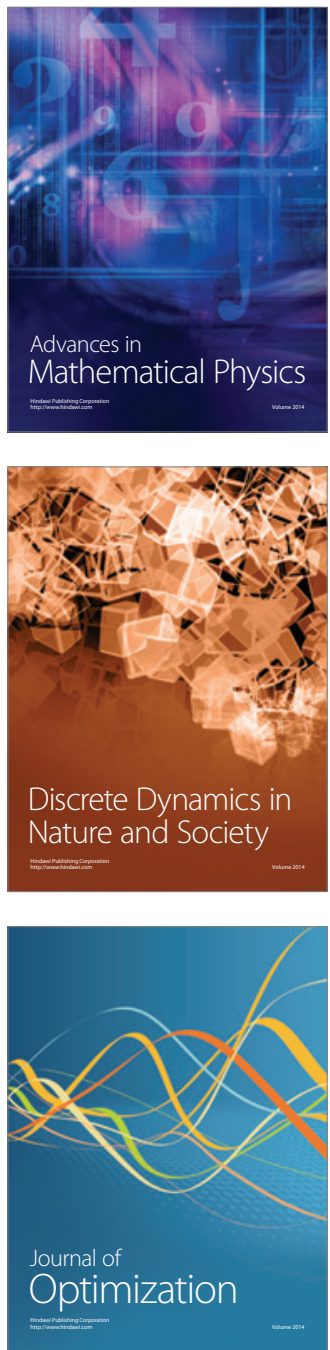